\title{
Heartbeat: Transcatheter procedures for secondary mitral regurgitation?
}

The development of transcatheter procedures to reduce the severity of mitral regurgitation (MR) provides a therapeutic alternative for patients with severe symptomatic primary MR who are at high risk for surgical intervention. It is less clear whether transcatheter mitral valve procedures are beneficial in patients with MR secondary to left ventricular (LV) systolic dysfunction or ischaemic heart disease. In a meta-analysis of 9 studies, including 2615 patients treated with a transcatheter mitral valve clip, Chiarito and colleagues ${ }^{1}$ found that patients with secondary MR had lower rates of mitral valve re-intervention at 1 year compared with those with primary MR (4\% vs $10 \%$; RR 0.60 ; 95\% CI: 0.38 to 0.97$)$. Residual MR was $2+$ or less in severity in most patients with either primary or secondary MR (58\% vs $54 \%$ ), with no differences in mortality, or device complications at 1 year (figure 1 ). However, secondary MR was associated with higher rates of persistent severe heart failure $(16 \%$ vs $8 \%)$ and heart failure re-hospitalisation (23\% vs $14 \%$ ) compared with patients with primary MR.

In an accompanying editorial, Orban and Hausleiter ${ }^{2}$ remind us that primary and secondary MR are due to markedly different disease processes. With primary MR, the valve leaflets themselves are abnormal so it seems plausible on physiological grounds that reducing MR severity might improve outcomes. In contrast, with secondary MR, backflow across the mitral valve is simply a secondary consequence of LV systolic dysfunction, with the underlying LV disease being the main determinant of clinical outcome. 'The cornerstone of treatment in this patient population is optimal pharmacotherapy and, if applicable, cardiac resynchronisation therapy. In end-stage heart failure, heart transplantation can be performed or further mechanical circulatory support

Correspondence to Professor Catherine M Otto, Division of Cardiology, University of Washington, Seattle WA 98195, USA; cmotto@uw.edu

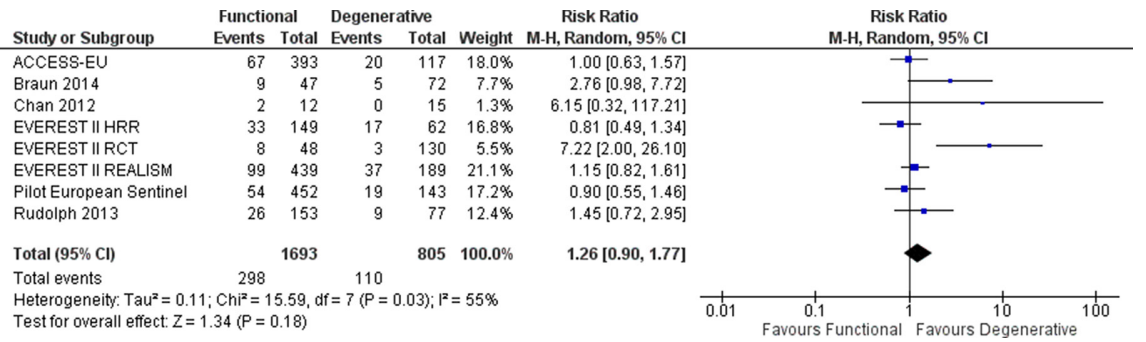

Figure 1 Risk estimates of overall mortality for functional mitral regurgitation versus degenerative mitral regurgitation. Forest plot shows results for overall mortality at 1 year. EVEREST II, Endovascular Valve Edge-to-Edge Repair Study II; RCT, randomised controlled trial.

can be provided with ventricular assist devices.' However, clinical experience suggests that transcatheter mitral valve procedures reduce heart failure
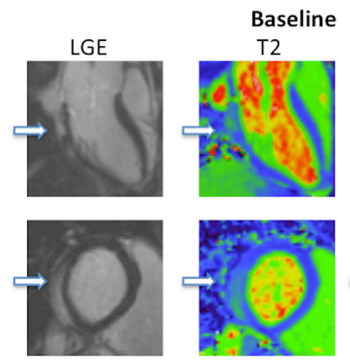

24h Post USPIO R2*
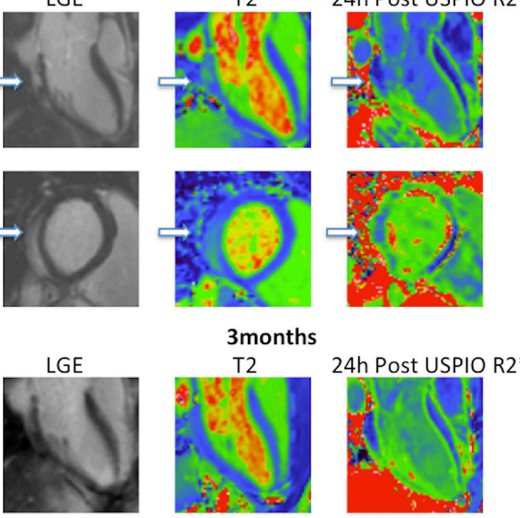
3months
T2 24h Post USPIO R2*
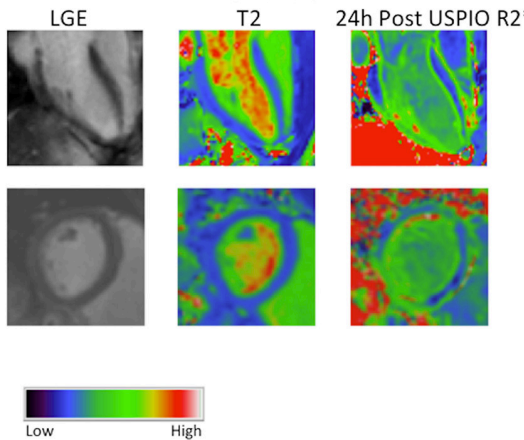

Figure 2 Images of patient with myocarditis. Three chamber and basal short-axis images of a patient with myocarditis displaying patchy posterolateral late gadolinium enhancement (LGE) (white regions, arrowed) that correspond to oedematous regions of myocardium on the T2 map (lighter regions, arrowed). There is no uptake of ultrasmall superparamagnetic particles of iron oxide (USPIO) in the postUSPIO R2* maps; corresponding regions may even have lower $\mathrm{R} 2$ * in this patient. Myocardial oedema appears to normalise but subtle LGE remains at 3 months. symptoms in some patients. Clinical trials of are in progress that will provide data on whether this procedure also improves clinical outcomes in patients with secondary MR.

Diagnosis of acute myocarditis is challenging but has improved with the availability of cardiac magnetic resonance (CMR) imaging for myocardial fibrosis and oedema. In this issue of Heart, Stirrat and colleagues ${ }^{3}$ tested the hypothesis that using ultrasmall superparamagnetic particles of iron oxide (USPIO)-enhanced CMR to detect myocardial macrophage activity provide additive diagnostic value in patients with suspected acute myocarditis. Compared with 10 normal volunteers, 14 patients with suspected myocarditis showed great myocardial oedema associated with late-gadolinium enhancement, as expected, but there was no difference in USPIO enhancement (figure 2). The authors conclude: 'This suggests that tissue-resident macrophages do not provide a substantial contribution to the myocardial inflammation in this condition.'

Mavrogeni and colleagues ${ }^{4}$ put this study into perspective by pointing out that CMR imaging has transformed diagnosis of myocardial disease. 'Pathophysiological phenomena such as oedema, endothelial dysfunction and patchy fibrosis that once only existed as scientific imagination became overt, measurable and available to clinicians for assessment.' Although the current study results are disappointing, 'USPIO-enhanced T2* imaging still 
holds promise as a non-invasive tool for the monitoring of tissue macrophages in the heart, especially in myocardial infarction, autoimmune myocarditis and acute transplant rejection.' They conclude: 'that the future of diagnostic imaging in myocardial inflammation will be enriched by various complementary approaches including multiparametric CMR, USPIO-enhanced T2* imaging and positron emission tomography (PET). This combination will hopefully allow us to always keep our head in the clouds, even though our feet are currently on the earth.'

Another interesting paper in this issue supports the concept that the simple measurement of blood pressure is an inadequate descriptor of vascular function. Ischikawa and colleagues $^{5}$ measured arterial stiffness and computed tomographic coronary artery calcification (CAC) in 320 patients without hypertension. Increased arterial stiffness, defined as a brachial-ankle pulse wave velocity (baPWV) of $18 \mathrm{~m} / \mathrm{s}$ or higher, was associated with higher CAC scores and with a higher rate of adverse cardiovascular events (a composite of cardiac death, myocardial infarction or revascularisation, and hospital admission for heart failure or stroke) (figure 3). Although measurement of pulse wave velocity is not yet ready for routine clinical use, accumulating data suggests that more complex parameters of vascular function than blood pressure alone-such

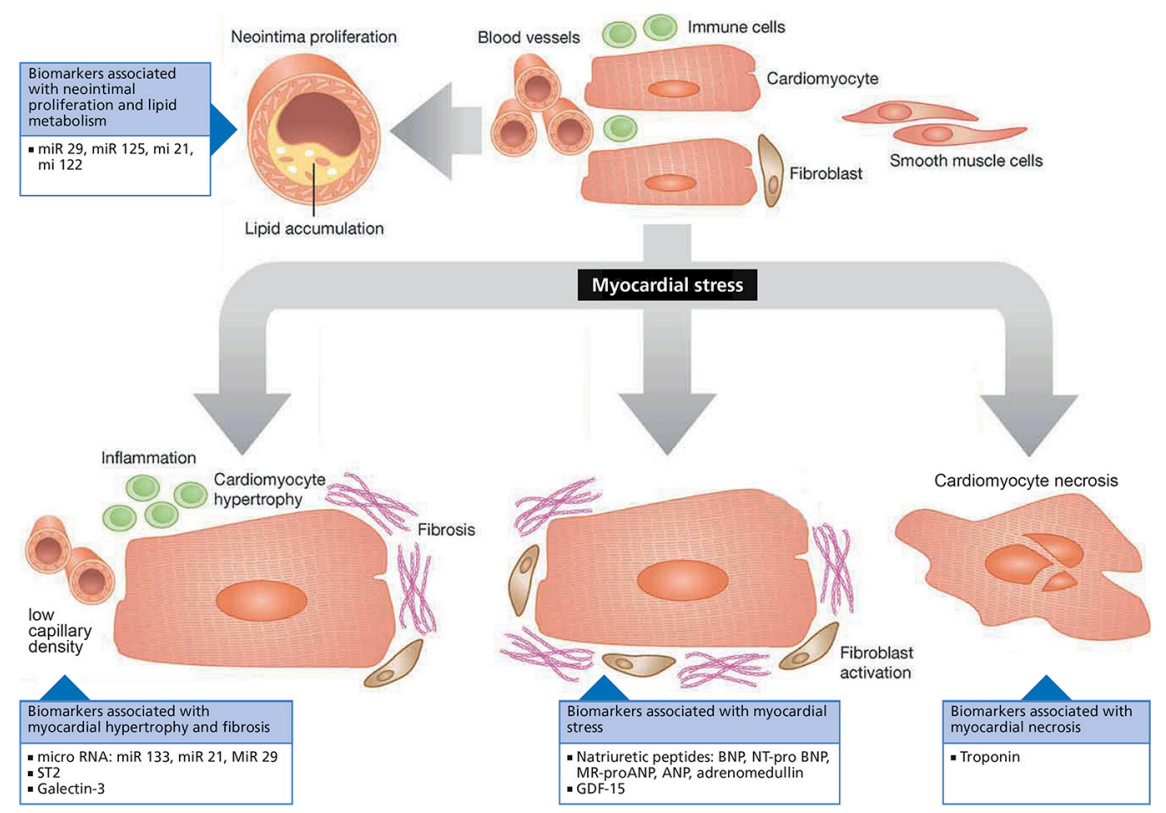

Figure 4 Serum biomarkers and their role in pathophysiology of VHD, adapted from Thum et al (EMBO Mol Med 2012;4:3-14). BNP, B-type natriuretic peptide; GDF-15, growth differentiation factor-15; MR-proANP, midregional proatrial natriuretic peptide; ANP, atrial natriuretic peptide; NTproBNP, N-terminal pro-B-type natriuretic peptide; VHD, valvular heart disease.

as pulse pressure, compliance, stiffness and pulse wave velocity-may provide additional prognostic information and might be useful in guiding medical therapy in the future.

The Education in Heart article summarises the pathophysiology and clinical utility of elevated serum biomarkers in patients with valvular heart disease. ${ }^{6}$ Numerous studies have

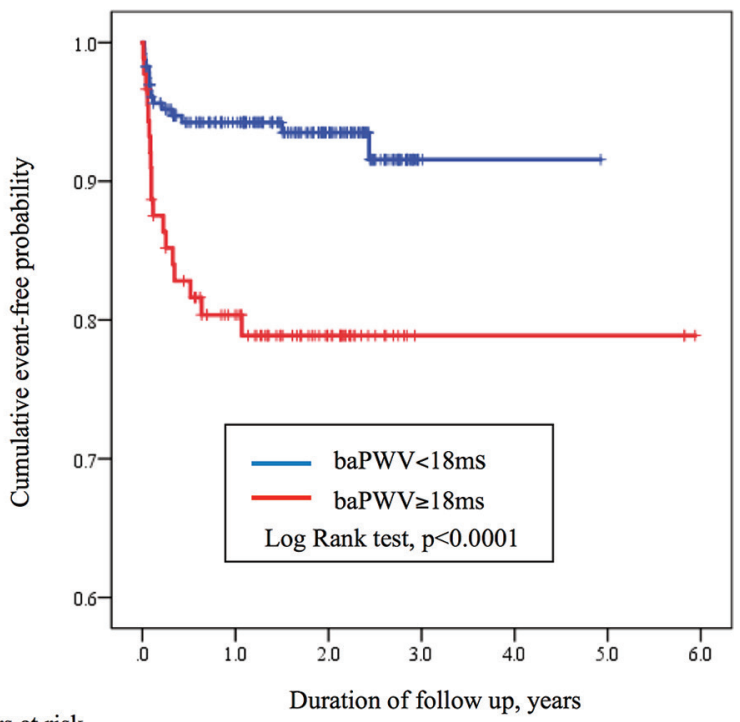

Numbers at risk

$\begin{array}{lccccc}\text { baPWV }<18 \mathrm{~ms} & 231 & 169 & 92 & 2 & 1 \\ \text { baPWV } \geq 18 \mathrm{~ms} & 89 & 57 & 28 & 3 & 3\end{array}$

Figure 3 Kaplan-Meier curve showing freedom from a cardiovascular event according to brachial-ankle pulse wave velocity (baPVW) group. shown that serum biomarkers are helpful for monitoring disease activity, prediction of disease progression, and identification of patients who are less likely to benefit from high-risk interventions. From the research perspective, these biomarkers may provide insights into the mechanisms of disease initiation and progression. Currently, many clinicians monitor serum B-type natriuretic peptide and troponin levels in selected patients with valvular heart disease, For the future, several other potential biomarkers also show promise (figure 4).

The Image Challenge in this issue shows a complication after endocardial radiofrequency ablation for ventricular tachycardia in a patient with a left ventricular assist device. ${ }^{7}$ See if you agree with the author's diagnosis.

Competing interests None declared.

Provenance and peer review Commissioned; internally peer reviewed.

(C) Article author(s) (or their employer(s) unless otherwise stated in the text of the article) 2018. All rights reserved. No commercial use is permitted unless otherwise expressly granted.

\section{Check for updates}

To cite Otto CM. Heart 2018;104:275-277.

Published Online First 15 January 2018

Heart 2018:104:275-277.

doi:10.1136/heartjnl-2018-312941 


\section{REFERENCES}

1 Chiarito M, Pagnesi M, Martino EA, et al. Outcome after percutaneous edge-to-edge mitral repair for functional and degenerative mitral regurgitation: a systematic review and meta-analysis. Heart 2018;104:306-12.

2 Orban M, Hausleiter J. Edge-to-edge mitral valve repair: solid data and a prosperous future. Heart 2018;104:280-1.
3 Stirrat CG, Alam SR, MacGillivray TJ, et al. Ferumoxytolenhanced magnetic resonance imaging in acute myocarditis. Heart 2018;104:300-5.

4 Mavrogeni SI, Markousis-Mavrogenis G, Kolovou G. Ferumoxytol in acute myocarditis: 'keep your head in the clouds and your feet on the earth'. Heart 2018;104:278-9.
5 Ichikawa K, Sakuragi S, Nishihara T, et al. Influence of arterial stiffness on cardiovascular outcome in patients without high blood pressure. Heart 2018;104:318-23.

6 Gardezi SK, Coffey S, Prendergast BD, et al. Serum biomarkers in valvular heart disease. Heart 2018;104:349-58.

7 Mangla A, Bjorklund JK, Kalra DK. An unusual echo after ventricular tachycardia ablation. Heart 2018;104:359-60. 